\title{
Aportes de la arqueología a la construcción de discursos sobre el pasado local desde la escuela. El caso del Pucara de Tilcara, Jujuy, Argentina
}

\author{
Mónica Montenegro*, María Elisa Aparicio**, Clarisa Otero*** y \\ María Clara Rivolta****
}

Arqueología

\begin{abstract}
* Instituto Interdisciplinario Tilcara, Facultad de Filosofía y Letras, Universidad de Buenos Aires. Centro Regional de Estudios Arqueológicos, Universidad Nacional del Jujuy; E-mail: mmontene@ucn.cl, ${ }^{* *}$ Instituto Interdisciplinario Tilcara, Facultad de Filosofía y Letras, Universidad de Buenos Aires; E-mail: meaparicio1@ hotmail.com, ${ }^{* * *}$ Instituto Interdisciplinario Tilcara, Facultad de Filosofía y Letras, Universidad de Buenos Aires; E-mail: clarisaotero@yahoo.com.ar, ${ }^{* * * *}$ Instituto Interdisciplinario Tilcara, Facultad de Filosofía y Letras, Universidad de Buenos Aires. Universidad Nacional de Salta; E-mail: clararivolta@hotmail.com
\end{abstract}

\begin{abstract}
Resumen
Este trabajo refiere a experiencias de arqueología pública realizadas en instituciones escolares de la Quebrada de Humahuaca, que contribuyeron a visibilizar el rol de la arqueología en la construcción del pasado local.

Nuestro objetivo ha sido observar apropiaciones y significados acerca de un sitio arqueológico local, en relación con dinámicas de construcción de patrimonio e identidades, que han venido gestándose en el marco de la implementación de políticas de multiculturalismo de Estado en la región.

Para ello, desarrollamos experiencias educativas que buscaron acercar la arqueología a la escuela primaria. De este modo, generamos espacios multivocales de re-construcción de conocimientos acerca de la disciplina arqueológica y su contribución a la comprensión del pasado local.
\end{abstract}

Palabras Clave: arqueología; comunidad educativa; interculturalidad; pasado local.

Contributions of archeology to the construction of discourses about the local past from school. The case of the Pucara de Tilcara, Jujuy, Argentina.

\begin{abstract}
This paper refers to some experiences of public archaeology done at Quebrada de Humahuaca's educative institutions which help us to visibility archeology's role in the construction of local past.

Our objective was to observe appropriations and significations about of an archaeological local site in relation to socials' dynamics of construction of heritage and identities, which have done in the farmer of Estate multiculturalism politics in this region.

For this purpose, we generate multivocals' spaces for re-construction of knowledges about the archaeological discipline and this contribution to the understanding of local past.
\end{abstract}

Keywords: archaeology; educative-community; inter-culturality; local past.

El trabajo que presentamos se desarrolla en un sector de los Andes Centro Sur. Precisamente, en el extremo septentrional del Noroeste Argentino, en la provincia de Jujuy (Figura 1). La misma, limita al Norte con la República de Bolivia, al Oeste con las Repúblicas de Chile y Bolivia y al este y sur con la provincia de Salta. Sobre una extensión de alrededor de $53.200 \mathrm{Km}^{2}$, despliega una interesante geografía, que ha dado lugar a cuatro diferentes regiones: Puna, Quebrada, Valles y Yungas. Las mismas se diferencian altitudinalmente, desde los 4500 msnm en la Puna hasta los 300 msnm en el extremo NE de la provincia, lo que provoca una gran diversidad de paisajes y recursos. Posee una ubicación geopolítica estratégica ya que se encuentra emplazada en área de triple frontera: Argentina-Bolivia-Chile, siendo por ello un espacio altamente dinámico y complejo a nivel cultural, donde las interacciones generan procesos de construcción de patrimonio e identidades (Montenegro y Aparicio 2008).

Este sector de la República Argentina, se ha convertido en los últimos años en escenario privilegiado para la implementación de programas de patrimonialización promovidas desde el Estado. Así, este territorio se ha 


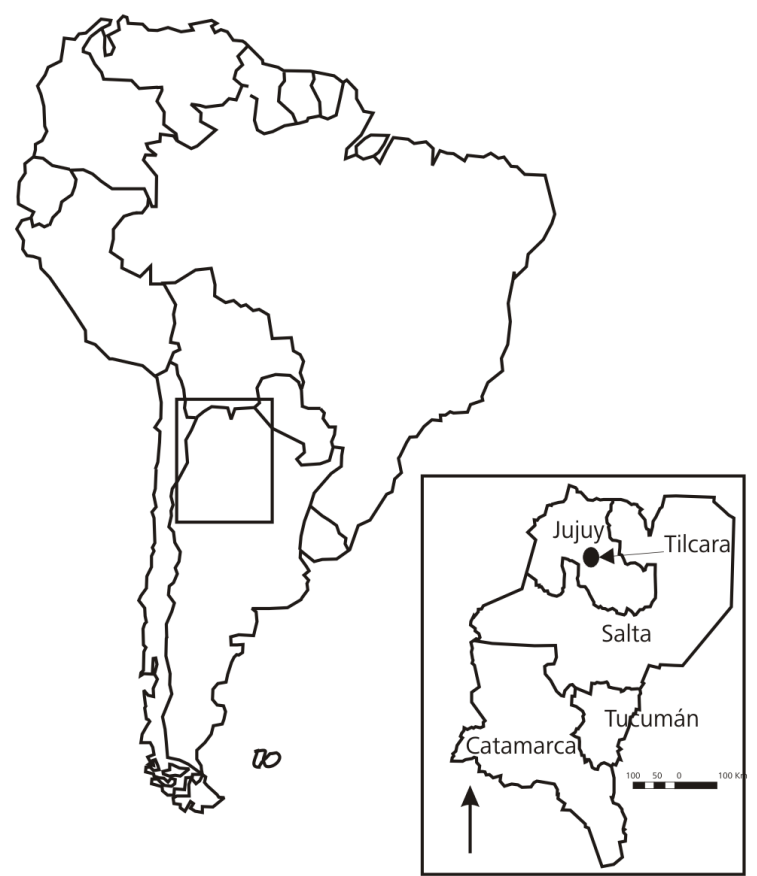

Figura1. Ubicación geográfica del área de investigación en el contexto Sudamericano.

Figure 1: Location of research area in the Southamerican context.

convertido en un espacio complejo donde los diversos grupos sociales desarrollan tramas de interacción política, económica y cultural en un intento por acceder a la propiedad del patrimonio. Esta interrelación genera, como sugieren algunos investigadores, fenómenos de confrontación y entrelazamiento; dichas prácticas contienen una dimensión cultural profunda, ya que algunos sectores comienzan a apropiarse con mayor énfasis, de elementos culturales locales para revitalizar su identidad (García Canclini 2006).

En este devenir, algunos sitios arqueológicos comenzaron a ser reapropiados por los pobladores ${ }^{1}$. Así, surgieron nuevas formas de interacción entre la comunidad local y los bienes arqueológicos, que motivaron serias diferencias; algunas contradicciones y paradojas en relación con valoraciones y apropiaciones de los bienes, han ido generando al interior de las comunidades disensos y re-posicionamientos sociopolíticos. En ocasiones el respeto y el resguardo de los "antigales" como espacios sagrados que promueven algunos grupos, se contrapone a la re-apropiación de los bienes arqueológicos, como posibles "recursos económicos" asociados al turismo, promovidos por otros sectores sociales; en esta dinámica, también participan los arqueólogos, asumiendo que sitios

\footnotetext{
1 La apropiación de sitios y bienes arqueológicos en Jujuy constituye un fenómeno social dinámico que muestra diferentes aristas y encierra diversas prácticas discursivas. Históricamente este proceso se dio en dos contextos diferentes: uno "estatal-manifiesto", ligado a la producción académica que impulsó el conocimiento de los vestigios materiales de las culturas del pasado; y otro "local-latente", que mantuvo una apropiación simbólica relacionada con el culto a los antepasados o Antigales (Montenegro 2010).
}

y materiales arqueológicos son evidencias materiales de desarrollos culturales del pasado, y por lo tanto los definen como "registro arqueológico". Esto genera rupturas inter-culturales, entre saberes ancestrales, discursos políticos y conocimientos científicos que no logran encontrar un espacio de diálogo. En esa dinámica, la práctica arqueológica es interpelada, y de cierto modo, se ve obligada a re-configurar sus vínculos con la comunidad local².

A nivel global estas problemáticas han sido suficientemente analizadas, y han conllevado no solo profundas reflexiones a nivel disciplinar, sino también posicionamientos ideológicos y políticos de los arqueólogos, que trascienden el espacio de las excavaciones y el laboratorio (Layton 1989; Ucko 1989; Trigger 1996; Hodder 2008). Especial interés revisten los estudios que apuntan a descentrar el discurso hegemónico en torno a la Arqueología y su práctica, que han tenido una intensidad creciente durante las últimas décadas del siglo XX. De cierto modo, los nuevos planteos que surgieron en respuesta a los fenómenos de globalización trajeron aparejados abordajes vinculados a la multivocalidad ${ }^{3}$. En tal sentido, Hodder (1999) sostiene que la arqueología no ha estado ausente de esos grandes procesos, dado que puede articular y promover la consolidación de identidades de comunidades que históricamente fueron segregadas y marginalizadas, y permitir que las mismas efectúen interpretaciones acerca del pasado, utilizando sus propios términos y aproximaciones a fin de construir significados diferentes a los modelos dominantes.

En este contexto, se ha vuelto necesario re-pensar la retórica discursiva en torno al rol de la arqueología en la construcción del pasado, el patrimonio arqueológico y las identidades locales. Un campo disciplinar que ha surgido como alternativa es la arqueología pública, y uno de los espacios de intervención, la educación (Merriman 2004). Sin embargo, llevar la arqueología al aula ha significado un proceso complejo (Schadla-Hall 2004; Stone y Molyneaux 1994); experiencias significativas son las que han desarrollado Högberg en escuelas de Suecia (2007), y Fernández Murillo (2003) para Bolivia. En Argentina, el primer trabajo que analizó la relación entre arqueología y educación fue el de Irina Podgorny (1994), aunque el mismo se restringió a la inclusión de la arqueología pampeana en la educación argentina. Otras publicaciones que acercaron elementos de arqueología regional a espacios de educación formal fueron el de María Esther Albeck y colaboradores (1996), realizado específicamente para la Quebrada de Humahuaca y

\footnotetext{
2 Uno de los casos más recientes, y que tomó estado público es el que protagonizó la Comunidad de Juella (Dpto. Tilcara), que interpeló a un equipo de investigaciones arqueológicas durante la campaña realizada al sitio Pucara de Juella y esto conllevó una serie de acciones destinadas a iniciar diálogos y negociaciones de acceso al campo; para mayor detalle ver publicación del Diario Pregón Año XIII, № 5542.

3 la cual entendemos en términos amplios como "la idea de que existen siempre múltiples interpretaciones del pasado" (Kojan, 2008: 75)
} 


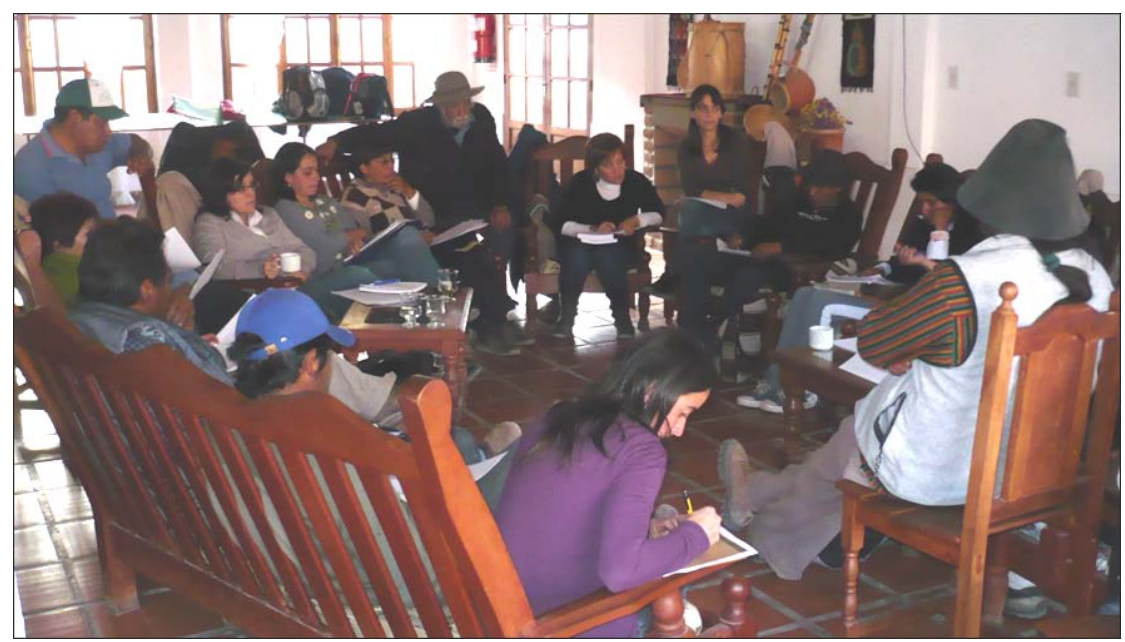

Figura 2. ESPAC 2010- Mesa Arqueología y Educación.

Figure 2. ESPAC 2010 - Archaeology and education roundtable.

los trabajos de Rossana Ledesma (2006.a y b), para la provincia de Salta.

En nuestro caso, el re-pensar la propia práctica arqueológica motivó un sinnúmero de interrogantes que tratamos de ir respondiendo a través de la conformación de grupos de trabajo. Apostamos a generar espacios no solo interdisciplinarios sino multivocales, donde los distintos actores que conformamos las comunidades locales de la Quebrada de Humahuaca pudiéramos re-conocernos y comenzar a escucharnos; fue así que durante 2009 y 2010 organizamos desde el Instituto Interdisciplinario Tilcara un encuentro anual denominado "Encuentro sobre práctica arqueológica y comunidades ESPAC (Figura 2).

Las prácticas discursivas de las comunidades en relación a la arqueología evidenciaron la larga trayectoria que tiene esta ciencia en la Provincia de Jujuy, y fueron asociadas en general a la intervención que tuvo la Universidad de Buenos Aires desde principios de siglo en el Pucara de Tilcara. Asimismo, quedó claro que el vínculo arqueólogos y comunidades locales ha transcurrido en una sucesión de "encuentros y desencuentros" traspasados por una variedad de contextos donde el Estado, cada poblado y/o comunidad en particular y, la propia acción profesional, fue cambiando en forma contrastante ${ }^{4}$. Así la arqueología era vista como una ciencia que tomaba elementos de las culturas "nuestras" para hacer "otros" discursos acerca del pasado; en esa dinámica, el Pucara de Tilcara experimentó diversas apropiaciones y significaciones por parte de los diferentes actores sociales nacionales, provinciales y locales.

\footnotetext{
4 En ciertos casos, los propios pobladores andinos fueron posicionados con criterios muy diversos y hasta antagónicos por parte de los arqueólogos. La injerencia del Estado Nacional se diversificó entre la construcción de un pasado ficticio y una intervención con escaso compromiso en relación a los bienes patrimoniales vinculados a la historia prehispánica. El pasado tuvo un discurso único y oficial donde se invisibilizaron la mayoría de las evidencias materiales del pasado prehispánico, que pasaron a formar parte de una otredad/no nacional (Montenegro y Rivolta 2010).
}

\section{El Pucará de Tilcara como monumento a la arqueología argentina}

Las primeras excavaciones arqueológicas en el Pucara de Tilcara comenzaron en 1908, cuando desde el Museo Etnográfico de la Facultad de Filosofía y Letras (FFyL), de la Universidad de Buenos Aires, se impulsaron las investigaciones en la región. Estos trabajos estuvieron dirigidos por Juan Bautista Ambrosetti, quien en aquel entonces era el Director del Museo. Su discípulo Salvador Debenedetti también participó en los trabajos de campo y, a la muerte de su maestro en 1917, continuó con las investigaciones en el Pucara hasta su fallecimiento a fines de la década de 1920. No obstante, estas investigaciones desarrolladas a través de la FFyL, las primeras referencias al Pucara se deben al arqueólogo sueco Eric Boman, quien en su publicación de 1908, sobre los resultados de la expedición de 1903 a la Quebrada de Humahuaca y Puna jujeña hace una breve mención del sitio (Otero 2009; 2010).

Más allá del carácter científico de estas primeras exploraciones, desde 1910 se planteó una mirada diferente del Pucara. En ese año, durante el desarrollo de la tercera campaña arqueológica, Debenedetti propuso la restauración del antiguo poblado con la idea de brindar un atractivo a los investigadores que concurrirían al XVII Congreso Internacional de Americanistas, que se realizaría ese año en Buenos Aires. Desde allí se había organizado un viaje en ferrocarril para conocer Tiwanaku, pasando por la Quebrada de Humahuaca. En este contexto, el Pucara de Tilcara se presentaría como una importante manifestación de los desarrollos culturales de Argentina.

Por otro lado, Debenedetti entendía que el sitio, también podría contribuir a los fines didácticos de la disciplina; pero sobre todo consideraba que aportaría al desarrollo económico de Tilcara, ya que se convertiría en un atractivo espacio de recreación para los numerosos turistas, que año tras año llegaban en mayores proporciones aprovechando el ferrocarril (Otero 2010). La repercusión de la restauración fue tan grande que incluso logró que 
se presentara un proyecto de ley para su financiación en la Cámara de Diputados de la Nación (Casanova 1968).

A su muerte, el carácter de esta empresa se trasmitió a su discípulo, Eduardo Casanova, quien no sólo continuó las tareas de restauración del sitio, sino que además organizó una Comisión de Homenaje a sus maestros, a través de la construcción de un monumento en la cima del Pucara. Para ello, contrató al reconocido arquitecto Martín Noel quien diseñó, emulando a los templos mesoamericanos, una pirámide trunca de cuatro caras sobre una gran plataforma, que a su vez presenta una importante escalinata para acceder a la cara frontal del monumento. Alí se encuentra ubicada la placa recordatoria que refleja la presencia en este espacio de una arqueología de base colonial; la misma contiene la siguiente leyenda:

\section{"De entre las cenizas milenarias de un pueblo} muerto

Exhumaron las culturas aborígenes dando eco al silencio"

La placa está emplazada en el sector central de la pirámide, enmarcada por dos estructuras de piedra de sobre-relieve, simulando los ingresos de los templos mayas. Asimismo, las ventanas ubicadas en el frente del monumento, sin llegar a ser trapezoidales, recuerdan las aberturas encontradas en importantes sitios incaicos de los Andes centrales (Figura 3). En el diseño de este monumento se pretendió materializar el imaginado estilo "arquitectónico nacional", promovido por los intelectuales de la época como Ricardo Rojas, y particularmente Noel, en el que se distinguía el uso de características artísticas y arquitectónicas de las grandes civilizaciones precolombinas; pero que nada tenían que ver con las características constructivas prehispánicas de los sitios de la Quebrada de Humahuaca (Otero 2010). El monumento, que se inauguró en 1935, fue el fiel reflejo de aquella ideología nacionalista, que "invisibilizó" a las culturas prehispánicas, en aras de la construcción de una "identidad nacional argentina".

Aunque la comunidad educativa local estuvo vinculada al devenir de la arqueología local, sólo concurría a visitar el sitio como un espacio educativo, el cual permitía reforzar la identidad argentina en esta área de frontera. Allí, donde era necesario definir los límites territoriales entre argentinos y bolivianos, fue importante instalar un mástil con una enorme bandera argentina (Casanova 1968), para que flameara significando e identificando la soberanía de la nación sobre cualquier desarrollo cultural anterior. No obstante, no era tan necesario estudiar el pasado prehispánico local en la escuela, no era significativo para los grandes desarrollos que se planteaban desde el moderno estado nacional. Las sociedades prehispánicas y su historia eran prácticamente desconocidas para la escuela; los arqueólogos excavaban y publicaban los resultados de sus investigaciones en importantes eventos científicos, que no llegaban a ser accesibles para la comunidad local; en consecuencia, desde la escuela, se operaba una de-construcción del pasado local.

\section{El Pucará como patrimonio arqueológico}

Hacia los primeros años de este nuevo milenio, desde el Estado Nacional y Provincial, comenzaron a efectuarse gestiones desde un proyecto que aparentemente ostentaba un modelo participativo de gestión ${ }^{5}$, que derivaron en la incorporación de una región de la provincia: "La Quebrada de Humahuaca" a la lista de Patrimonio Mundial de la UNESCO, en el año 2003. Los discursos oficiales sostenían que estas acciones podrían servir para atenuar la seria crisis económica que enfrentaba la provincia6. Para ello se promovieron activaciones patrimoniales ${ }^{7}$, que redundaron en la explotación de los bienes culturales como potenciales recursos de desarrollo económico asociados al turismo, reconociéndolos como parte del patrimonio provincial. Finalmente sucedió lo que señalan algunos investigadores: el patrimonio comenzó a considerarse un capital cultural, que se acumula, se reconvierte, produce rendimientos y es apropiado de distinta manera por los diversos sectores; (Rosas Mantecón 1999; García Canclini 2001, 2006).

Este reconocimiento, asociado vertiginosamente a programas de desarrollo turístico, generó: una re-conceptualización del patrimonio como fuente de ingresos económicos. En ese contexto, las comunidades comenzaron a re-apropiarse y resignificar bienes arqueológicos como parte de su patrimonio, construyendo una realidad social compleja, que se ha ido re-semantizando con el transcurrir del tiempo; en consecuencia, el mismo patrimonio que en el pasado fuera desvalorizado, escondido u olvidado a causa de las políticas emanadas desde el Estado nacional, en el presente se descubre como un mecanismo de memoria ligado a la identidad; como marcador territorial, en

\footnotetext{
5 De acuerdo con Belli y Slatvutsky (2005: 49), "para desarrollar este proyecto con bajos niveles de conflictividad en un espacio con tantas desigualdades se creó un modelo de intervención con soporte "participativo", donde participación refiere más a prestar conformidad, una suerte de consenso acordado en el que los actores exhibían el libreto aportado desde las instituciones oficiales, para lo cual los participantes eran, en la medida de los posible, seleccionados".

6 Algunos autores han sugerido que el patrimonio, como construcción social, posee una capacidad para representar simbólicamente una identidad, hecho que explica, por qué se movilizan recursos para su puesta en valor y protección. Los símbolos patrimoniales, resultado de la relación entre ideas y valores de una sociedad, son dinámicos, en tal sentido, la correlación entre intereses, valores y situaciones históricas cambiantes, permiten entender ciertas gestiones patrimoniales como estrategias políticas (Prats 1997).

7 Según Prats (1997), las activaciones patrimoniales son sistemas de símbolos que actúan para suscitar entre los miembros de una comunidad motivaciones y disposiciones poderosas, profundas y perdurables, formulando concepciones de orden general acerca de la identidad de esa comunidad y dando a estas concepciones una apariencia de realidad tal que sus motivaciones y disposiciones parezcan emanar de la mas estricta realidad.
} 


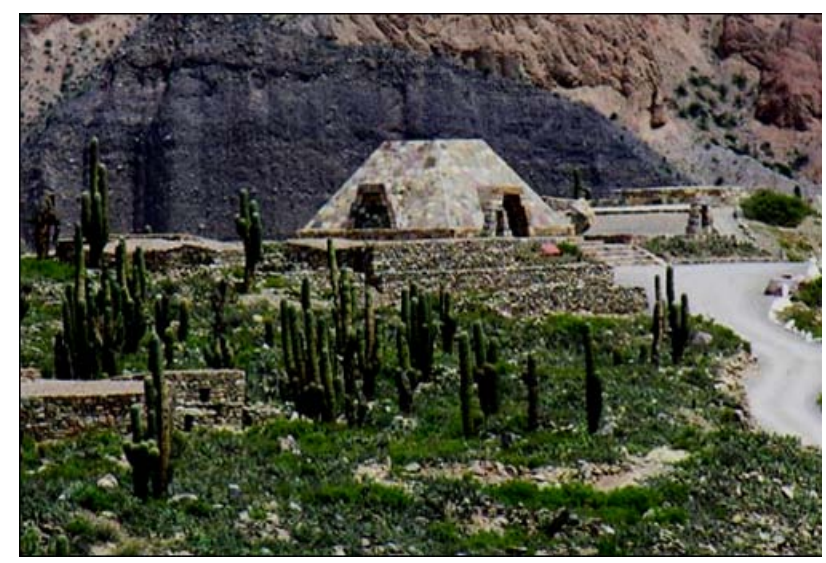

Figura 3. Vista del Pucara de Tilcara y su pirámide.

Figure 3. Pucará de Tilcara view showing the pyramid.

conflictos jurídicos por la propiedad de las tierras; o como potencial recurso de desarrollo económico asociado a diversos proyectos turísticos.

\section{Construcción de patrimonio arqueológico en la escuela}

En este contexto, nuestro interés se centró en conocer las dinámicas de configuración del patrimonio arqueológico en escuelas primarias de Tilcara y zonas aledañas. Definimos como unidades de observación a las siguientes escuelas: Escuela N 7 "Domingo F. Sarmiento" y Escuela Normal "Dr. Eduardo Casanova", de Tilcara; Escuela No 31 "Coronel Arias", de Huacalera; Escuela No 101 "Helguera Sánchez", de Colonia San José; Escuela N²41 "Provincia de Neuquén", de Juella. Las unidades de análisis fueron: directivos, docentes y alumnos de los establecimientos educativos mencionados. El trabajo de campo se realizó en diferentes contextos áulicos desde $4^{\circ}$ a $7^{\circ}$ grado y abarcó dos ciclos lectivos: 2009 y 2010. La selección de informantes, se operó a través de una "muestra de oportunidad", donde ponderamos la situación de encuentro, la capacidad de interpretar los objetivos de trabajo conjunto y las posibilidades de continuar la relación (sensu Guber 2004). Las actividades estuvieron orientadas a conocer y analizar la relación que se establecía entre patrimonio, arqueología y construcción del pasado local.

A los fines del presente trabajo solo presentaremos un diagnóstico acerca de los conocimientos previos que tenían los niños sobre arqueología y pasado local. Nuestro referente empírico se constituyó en base a discursos, prácticas y representaciones acerca del patrimonio y la arqueología, que registramos en la interacción cotidiana de los actores educativos. La modalidad seleccionada para obtener esos trabajos fue talleres de expresión grafo plástica, privilegiándose en todos los casos la participación de padres y abuelos a través de los relatos sobre las manifestaciones del pasado local. Siguiendo las propuestas didácticas de Leonor Spravkin (1997), planteamos temas centrales, pero dejamos en libertad a cada niño para que seleccione sus estrategias de trabajo y resoluciones técnicas, desarrollando su obra según su sentido estético y emotividad. Participamos de los talleres una arqueóloga, una antropóloga y el docente a cargo del grado, junto a los alumnos, obteniendo en estos espacios ciento veinte trabajos que luego fueron analizados.

Para comenzar preguntamos a los niños si conocían el Pucara de Tilcara, la mayoría (94\%) contestó afirmativamente. Los docentes señalaron que este sitio se estudia en 4to. grado, desde el área de Ciencias Sociales, cuando se aborda el tema: Período Indígena de Jujuy, y en esa oportunidad suelen realizar una visita al Pucara. Consultamos a los niños que habían visitado el sitio para saber que parte les había resultado más importante:

\begin{tabular}{ccc}
\hline La pirámide & Las casitas & La iglesia \\
\hline $75 \%$ & $16 \%$ & $9 \%$ \\
\hline
\end{tabular}

Tabla 1: ¿Qué parte del Pucara de Tilcara es la más importante?

Table 1. Which part of Tilcara Pucara is the most important?

La mayoría señaló a la pirámide como el sector más importante (Figuras 4 y 5). En general, las explicaciones estaban relacionadas con su ubicación (en la cima), con su morfología, diferente de todas las demás construcciones (piramidal), con el tipo de construcción (según los niños estaba mejor construida), con su tamaño (es el edificio más alto y puede verse desde la ruta).

\begin{tabular}{cccc}
\hline $\begin{array}{c}\text { Una construc- } \\
\text { ción de los in- } \\
\text { dios }\end{array}$ & $\begin{array}{c}\text { La pirámide } \\
\text { de los Incas }\end{array}$ & $\begin{array}{c}\text { Un monumento } \\
\text { de la arqueo- } \\
\text { logía }\end{array}$ & $\begin{array}{c}\mathrm{N} \mathrm{s} / \\
\mathrm{Nc}\end{array}$ \\
\hline $49 \%$ & $24 \%$ & $9 \%$ & $18 \%$ \\
\hline
\end{tabular}

Tabla 2: ¿Qué es la pirámide del Pucara?

Table 2. What is the pyramid of the Pucara?

Los niños, en su mayoría, consideraron que la pirámide era una construcción realizada por los antiguos habitantes del Pucara; para un segundo grupo, se trataba de una pirámide que realizaron los Incas cuando llegaron a la Quebrada de Humahuaca. Un tercer grupo afirmó no saber. Solo un número significativamente menor señaló que se trataba de un monumento relacionado con los primeros arqueólogos.

\begin{tabular}{llll}
\hline $\begin{array}{l}\text { Es un Antigal } \\
\text { grande }\end{array}$ & $\begin{array}{l}\text { Un lugar tu- } \\
\text { rístico }\end{array}$ & $\begin{array}{l}\text { Un lugar donde } \\
\text { trabajan los } \\
\text { arqueólogos }\end{array}$ & Ns/Nc \\
\hline $52 \%$ & $31 \%$ & $13 \%$ & $4 \%$ \\
\hline
\end{tabular}

Tabla 3. ¿Qué es el Pucara de Tilcara?

Table 3. What is the Pucará de Tilcara? 
La mayoría de los niños indicaron que el Pucara es un gran antigal. Un segundo grupo comentó que se trataba de un lugar turístico, al que llegan visitantes de todas partes del país; algunos comentaron que también llegan extranjeros, sobre todo desde la nominación de la Quebrada como Patrimonio de la Humanidad. Un tercer grupo señaló que es un lugar donde los arqueólogos desentierran cosas muy valiosas, parte de ellos incluso destacaron que los arqueólogos excavaban tesoros. Del total, fueron muy pocos los niños que dejaron esa pregunta sin responder.

\begin{tabular}{cc}
\hline Para poner en los museos & Ns/Nc \\
\hline $38 \%$ & $62 \%$ \\
\hline
\end{tabular}

Tabla 4. ¿Para qué se utilizan las cosas que desentierran los arqueólogos?

Table 4. What use is given to things unearthed by archaeologists?

Los resultados confirman la escasa información que tienen los alumnos de escuelas primarias de este sector de la Quebrada sobre el rol de la arqueología en relación con la construcción de discursos acerca del pasado, hecho que habíamos constatado en investigaciones previas en otros sectores de la Provincia de Jujuy (Montenegro 2009). Aunque en algunos casos, saben que una de las actividades de los arqueólogos es excavar, allí se agotan las explicaciones sobre nuestro rol profesional. Al parecer, aunque el Pucara de Tilcara es visitado en el marco de actividades escolares, resulta dificultoso para los niños asociar el trabajo de los arqueólogos con el estudio de las sociedades del pasado de la Quebrada de Humahuaca.

Por lo demás, hemos observado que existe, en la mayoría de los casos un conocimiento y significación del sitio como espacio sagrado, como "Antigal", un lugar que no debe ser perturbado porque puede traer enfermedades tanto psicológicas como físicas a quienes no lo respeten; los niños traen esos conocimientos desde sus hogares, pero no es frecuente que los mismos se expresen en el contexto áulico, ya que no se promueven espacios de participación. Por lo demás, no observamos que en la

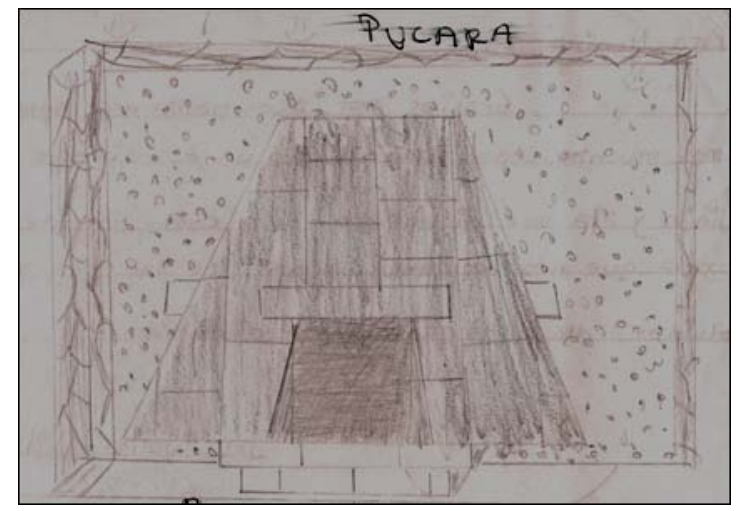

Figura 4. El Pucara. Representación de un alumno de $4^{\circ}$ de la escuela de Huacalera.

Figure 4. The Pucara. Huacaleras's 4th grade student representation. escuela se manifiesten fenómenos de re-conocimiento ni de apropiación del sitio arqueológico como parte del patrimonio cultural, ni de la identidad local. Los docentes nos han comentado que posiblemente esta situación se derive de la falta de tratamiento de esos temas en el contexto áulico; la inexistencia de contenidos de arqueología en el Diseño Curricular Oficial para Educación Primaria y la escasa formación de los docentes en temas relacionados con arqueología conlleva la imposibilidad de considerar esos temas en el aula. Esto nos lleva a considerar nuevas investigaciones que permitan indagar en forma profunda esos procesos socioculturales, en relación con el curriculum oculto.

\section{Reflexiones finales}

Los discursos muestran que, aunque en la provincia de Jujuy han venido desarrollándose importantes investigaciones arqueológicas durante un gran lapso temporal, esta ciencia pasa casi inadvertida para ciertos sectores de la comunidad local, que trabajan en relación con las evidencias materiales del pasado. Las representaciones de la comunidad educativa primaria resultan reveladoras en tal sentido, y en este punto asumimos la responsabilidad que nos cabe frente al desconocimiento social de nuestra disciplina.

Las respuestas despertaron nuevos interrogantes que nos movilizan a generar espacios multivocales, para la construcción de discursos sobre el pasado local. Sin embargo nos preguntamos: hasta que punto somos concientes que la "multivocalidad es una cuestión compleja, dado que su capacidad de empoderamiento es tanta como su capacidad de neutralización", como ha sugerido Cristóbal Gnecco (2002:18). Entendemos que es necesario seguir estudiando propuestas pedagógicas para trabajar temas arqueológicos en el contexto áulico, desde una perspectiva de interculturalidad crítica, asumiendo en la praxis una postura de de-colonialidad de conocimientos (Walsh 2009). Nuestra intención es acercar contenidos en forma organizada y en un lenguaje próximo

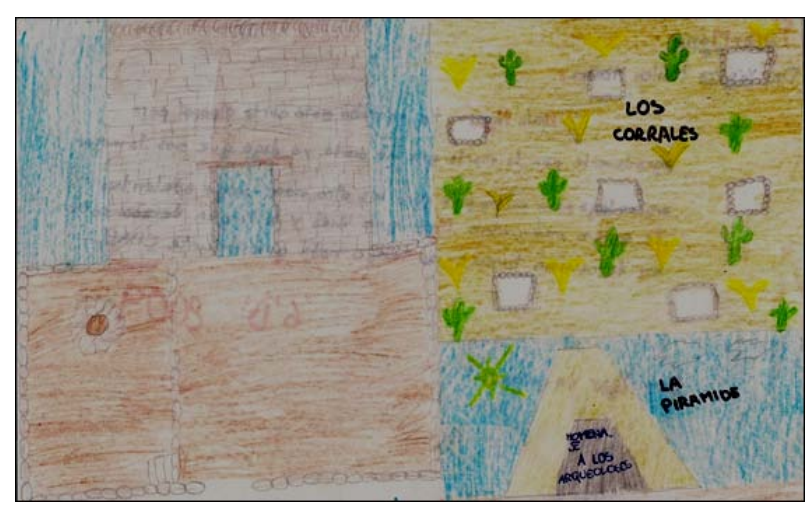

Figura 5. El Pucara. Representación de un alumno de $4^{\circ}$ de la escuela de Huacalera.

Figure 5. The Pucara. Huacaleras's 4th grade student representation. 
a las experiencias e inquietudes de los miembros de la comunidad educativa; esto puede concretarse a partir de escuchar lo que dicen y sienten los actores acerca de la historia local y regional.

Entendemos que estas propuestas podrían contribuir a la superación del uso irreflexivo de términos como "período indígena", "arqueología" y "pasado", en la comunidad educativa. También permitirían optimizar el uso del Pucara de Tilcara como herramienta didáctica para que los estudiantes puedan conocer los desarrollos culturales pretéritos. Para nosotras, significa asumir una mirada crítica sobre nuestro rol como arqueólogas en relación con la co-construcción de conocimientos, que pudieran ser apropiados por los alumnos, desde una mirada intercultural. En este escenario, consideramos a la enseñanza como una acción intencional de transmisión cultural que puede favorecer el desarrollo de capacidades, en relación al contexto social en el que se desarrolla.

Sin embargo, somos conscientes de que proponer espacios de construcción del conocimiento desde una perspectiva intercultural no es algo sencillo. Adherimos a lo señalado por Rubinelli (2010:40) que "la formación para la interculturalidad requiere una conjunción de distintos aportes desde perspectivas disciplinarias diversas y complementarias que permitan desarrollar miradas y análisis no convencionales, que favorezca procesos de construcción de concepciones éticas, antropológicas, epistemológicas que en un primer momento desestructuren las certezas acumuladas desde formaciones tradicionales". Para ello proponemos trabajar desde perspectivas educativas que se construyen con relación a otros sectores de la población, partiendo de la noción de pedagogías de "pensar con" y desarrollando un actuar pedagógico fundamentado en la humanización y descolonización. Esto significa no sólo ser conscientes de los patrones de poder coloniales que están presentes en nuestra sociedad, sino además de "asumir con compromiso una accionar dirigido a la transformación, la creación y a ejercer el proyecto político, social, epistémico y ético de la interculturalidad" (Walsh 2009:50)

Creemos que resulta útil analizar las prácticas discursivas de los distintos sectores de la comunidad acerca de la arqueología, los arqueólogos y el patrimonio arqueológico; discutiendo asimismo los alcances de esas nociones en relación con la re-configuración identidades y territorios. Por otra parte, resulta importante analizar el grado de participación que tiene la arqueología en las dinámicas de activación patrimonial que se promueven desde el Estado. Sostenemos la necesidad de renovar la mirada, de observar el aquí y ahora de la arqueología, que impone no solo una reflexión teórica, sino y fundamentalmente un posicionamiento sociopolítico en relación a los discursos que estamos construyendo en el presente sobre el pasado.

Tilcara, 27 de Julio 2010

\section{Agradecimientos}

A las comunidades educativas de la Quebrada de Humahuaca, que nos permitieron compartir espacios de co-construcción de conocimientos sobre el pasado local; especialmente a la Prof. Rosa Contreras, Delegada de Región II del Ministerio de Educación de la Provincia, y a los directivos y docentes: María Elizabeth Farfán de Ayarde, María Galán, Gabriela Soto, Mariana de La Riestra, y Ezequiel Chañi. Agradecemos a Andres Izeta, y a los evaluadores por sus amables sugerencias que sirvieron para enriquecer el trabajo. Ninguno de ellos es responsable de las expresiones aquí vertidas.

\section{Referencias Bibliográficas}

Belli, E. y R. Slavutsky 2005 Discursos patrimonialistas. Consecuencias prácticas. Belli, E. y R. Slavutsky (Eds), Patrimonio en el Noroeste Argentino: Otras historias, pp. 39-64. Instituto Interdisciplinario Tilcara, Universidad de Buenos Aires.

Casanova, E. 1968. El Pucará de Tilcara. (Antecedentes, Reconstrucción, Guía). Publicación No.1. Facultad de Filosofía y Letras, Museo del Pucará de Tilcara, Universidad de Buenos Aires.

García Canclini, N. 2001. Culturas Híbridas. Estrategias para entrar y salir de la modernidad. Paidos, Buenos Aires.

García Canclini, N. 2006. Diferentes, Desiguales y Desconectados. Mapas de la interculturalidad. Gedisa Editorial, Barcelona.

Gnecco, C. 2002. La indigenización de las arqueologías nacionales. Convergencia Año 9, $N^{\circ}$ 27: 133-149, Universidad Autónoma del Estado de México, Toluca, México http://redalyc.uaemex.mx (02/08/2010).

Guber, R. 2004. El salvaje metropolitano. Reconstrucción del conocimiento social en el trabajo de campo. Paidos, Buenos Aires.

Hodder, I. 2008. Multivocality and Social Archaeology. Habu, J.; C. Fawcett y J. Matsunaga (Ed), Evaluating Multiple Narratives: Beyond Nationalist, Colonialist, Imperialist Archaeologies, 196-200. Springer, Berkeley.

Kojan, D. 2008. Paths of Power and Politics: Historical Narratives at the Bolivian Site of Tiwanaku. Habu, C. Fawcett and J.M. Matsunaga (eds), Evaluating Multiple Narratives. Beyond Nationalist, Colonialist, Imperialist Archaeologies. J. pp. 69-85, Springer.

Layton, R. 1989. Introduction: conflict in the Archaeology of living traditions. Layton, R. (Ed), Conflict in the Archaeology of the Living Traditions, Routledge, London \& New York. 
Merriman, N. 2004. Involving the public in museum archaeology. Merriman, N. (Ed.), Public Archaeology, pp. 85-108. Routledge, London y New York.

Montenegro, M. 2010. Patrimonio arqueológico en el sector septentrional del Noroeste Argentino. Propuestas pedagógicas para su preservación. Tesis para optar al título de Doctor en Antropología. Universidad Católica del Norte y Universidad de Tarapacá, Chile.

Montenegro, M. 2009. El patrimonio arqueológico de Jujuy: miradas diversas desde la escuela. Estudios Sociales del NOA, nueva serie 10: 107-121.

Montenegro, M. y M. E. Aparicio. 2008. Los niños y el patrimonio ¿Una deuda de la Educación? Cuadernos 34: 103-119.

Montenegro, M. y M. E. Aparicio. 2010. Festividades, celebraciones y patrimonio en Jujuy: experiencias desde la escuela. Rubinelli, M. L. (Comp.) ¿Los otros como nosotros? Interculturalidad y Ciudadanía en la Escuela. Reflexiones desde América Latina, Tomo II: 61-72. Unidad de Pensamiento Latinoamericano, relaciones interétnicas e interculturales, Universidad Nacional de Jujuy.

Montenegro, M. y M.C. Rivolta. 2010. Re-construyendo el presente desde el pasado: los arqueólogos y sus desafíos. Ponencia presentada al Simposio "Arqueología y Desarrollo", V Encuentro de Teoría Arqueológica de América del Sur. Universidad Central de Venezuela, Caracas.

Otero, C. 2009. Cien años de investigaciones. El Pucará de Tilcara cuenta su historia, Conferencia en el marco del II Taller Internacional de Arqueología del NOA y Andes Centro Sur, Facultad de Humanidades y Ciencias Sociales, Universidad Nacional de Jujuy.

Otero, C. 2010. La Arqueología en el relato oficial del Estado Nacional. Una mirada desde el Pucará de Tilcara (Jujuy, Argentina). Revista Arqueología Suramericana. Universidad del Cauca, Universidad Nacional de Catamarca y World Archaeological Congress. En prensa.

Prats, LL. 1997. Antropología y Patrimonio. Ariel, Barcelona.
Rosas Mantecón, A. 1999. Las jerarquías simbólicas del patrimonio: distinción social e identidad barrial en el Centro Histórico de la Ciudad de México. http://www. naya.org.ar/patrimonio (10/07/2008)

Rubinelli, M. L. 2010. Algunas cuestiones acerca de una educación intercultural. Rubinelli, M. L. (Comp.) ¿Los otros como nosotros? Interculturalidad y Ciudadanía en la Escuela. Reflexiones desde América Latina, Tomo II: 37-48. Unidad de Pensamiento Latinoamericano, relaciones interétnicas e interculturales, Universidad Nacional de Jujuy.

Schadla-Hall, T. 2004. The comforts of unreason: the importance and relevance of alternative archaeology. Merriman, N. (Ed.) Public Archaeology, pp. 255-271. Routledge, London y New York.

Spravkin, L. 1997. Educación plástica en la escuela. Un lenguaje en acción. Ediciones Novedades educativas, Buenos Aires.

Trigger, B. 1996. Alternative Archaeologies: Nationalist, Colonialist, Imperialist. Preucel, R. e I. Hodder (Eds.) Contemporary Archaeology in Theory, pp. 615-631. Blackwell Publishers, Oxford, UK.

Ucko, P. 1989. Foreword. Layton, R. (Ed) Conflict in the Archaeology of the Living Traditions. Routledge, London \& New York.

Walsh, C. Interculturalidad crítica y pedagogía decolonial: in-surgir, re-existir y re-vivir. Medina Melgarejo (Comp.) Educación intercultural en América Latina. Memorias, horizontes históricos y disyuntivas políticas. Universidad Pedagógica Nacional, Plaza Valdez Editores, México.

\section{Publicaciones periódicas}

Unidos para prevenir saqueos arqueológicos. Diario Pregón, San Salvador de Jujuy, Argentina, Año XIII, Nº 5542, Sección Interior - Tilcara. http://www.pregon. com.ar/vernoticia.asp?id=95835 